\title{
EFECTO DE INHIBIDORES DE PARDEAMIENTO Y LA TÉCNICA DE ATMÓSFERA MODIFICADA EN MANGO (Mangifera indica L) VARIEDAD VAN DYKE MÍNIMAMENTE PROCESADO
}

\section{EFFFFECT OF INHIBITORS AND THE TECHNIQUE OF MODIFIED ATMOSPHERE IN MANGO (Mangifera indica $L$ ) VARIETY VAN DYKE MINIMALLY PROCESSED}

\author{
Jesús Antonio Galvis Vanegas ${ }^{1}$, Martha Cecilia Castillo Muñoz ${ }^{2}$
}

\begin{abstract}
Resumen
El mango (Mangifera indica $L$ ) variedad Van Dyke es un fruto apetecido a nivel nacional e internacional por su agradable aroma, intenso color amarillo y buen balance de ácidos/azúcares.
\end{abstract}

La investigación se desarrolló en dos etapas. En la primera se determinó la respuesta de los trozos de fruto a la aplicación de tres inhibidores de pardeamiento en dos concentraciones: (ácido cítrico a 150 y 300 ppm; ácido ascórbico a 250 y 500 ppm y L-Cysteina a 100 y 200 ppm), con relación a la calidad y tiempo de almacenamiento; en la segunda, se estableció el tiempo de vida útil de modo que conserve sus características organolépticas, cuando se combina el mejor inhibidor de pardeamiento, con la técnica de atmósfera modificada en concentraciones de $5 \%$ de $\mathrm{CO} 2,5 \%$ de $\mathrm{O} 2$ y $90 \%$ de $\mathrm{N} 2$, empleando dos tipos de empaque: canastillas plásticas y bandejas de icopor, colocadas dentro de bolsas de polietileno de baja densidad (LDPE) calibre 2 (0.035 $\mathrm{mm}$ de espesor).

Los trozos de mango se almacenaron a temperatura de $3 \pm 1^{\circ} \mathrm{C}$ y humedad relativa (HR) entre 85 y $90 \%$, durante 9 días en la primera etapa y durante 12 días en la segunda.

Las variables seleccionadas para evaluar el comportamiento del producto durante todo el experimento fueron: Pérdida de peso, Sólidos solubles, pH, azúcares y al final de los experimentos se realizó un análisis sensorial. Las variables medidas fueron sometidas a análisis de varianza y la comparación de las medias entre tratamientos se hizo mediante la prueba de Tuckey a un nivel de significancia del 95\%. Se usó un diseño experimental completamente al azar con estructura factorial de 3 × 2 en la primera etapa y 2 × 2 en la segunda, muestras de 250 gramos en tres replicaciones fueron utilizadas para cada tratamiento.

El tratamiento con inhibidores que obtuvo las mejores respuestas organolépticas y físicas durante el almacenamiento fue el de trozos de mango tratados con ácido ascórbico en concentración

\footnotetext{
1 Ingeniero Agrícola, PhD Ciencias Agropecuarias Universidad Nacional de Colombia, Docente superior IV Fundación Universidad Agraria de Colombia UNIAGRARIA. E mail: jagalvisv@gmail.com

2 Ingeniera Agrícola, MSc Ingeniería Agrícola Universidad Nacional de Colombia. E mail: mccastillom@unal.edu.co
} 
de 500 ppm, por lo que en el almacenamiento en atmósfera modificada se empacaron trozos de mango tratados con este inhibidor en dicha concentración en dos condiciones de empaque obteniéndose la mejor respuesta en aquellos trozos de mango tratados con ácido ascórbico empacados en bandeja de icopor con una vida útil de 12 días.

Palabras claves: inhibidor de pardeamiento, mango, atmósfera modificada, conservación.

\section{Abstract}

The Van Dyke mango variety is a much desired fruit in the country and outside of it, thanks to its pleasant smell, its yellow and orange intense colour and sugar/acids good balance.

This research shows the effect of the application of browning inhibitor agents combined with modified atmosphere technique in Van Dyke variety mango minimally processed.

The project was developed in two parts. The first part was about determining into three browning inhibitor agents and two different concentrations: (citric acid in concentrations of 150 ppm and 300 ppm, ascorbic acid in concentrations of 250 ppm and 500 ppm and LCysteina in concentrations of $100 \mathrm{ppm}$ and $200 \mathrm{ppm}$ ), which was the one that showed the best reaction related to the quality and storage time.

The second part determined the shelf life of the mango minimally processed for keeping its organoleptics, characteristics when it combines the best browning inhibitor agents found in the first stage, using the modified atmosphere technique with $5 \%$ of $\mathrm{O}_{2}, 5 \%$ of $\mathrm{CO}_{2}$ and $90 \%$ of $\mathrm{N}_{2}$ and employing two different packaging conditions: plastic containers and polystyrene trays, put into bags of polyethylene-low density (LDPE) 2 calibre (0.035 $\mathrm{mm}$ of thickness).

The slices of mango were storage in $3 \pm 1{ }^{\circ} \mathrm{C}$ and with an HR between 85 and $90 \%$ during nine days in the first stage and twelve days the second part. In the first part, some processed fruit but not immersed in any of the solutions was taken as proof in the second part, some processed but not treated with browning inhibitor packed in the same conditions in modified atmosphere was taken as proof.

The variables choose to see the behaviour of the packed product in each experimental stage was: lost of weight, $\mathrm{pH}$, brix degrees, sugars: sucrose, fructose and glucose.

The SAS statistics program was used to analyze the changes of the properties through the time, as well as its answer depending the type of the treatment (first stage) and the condition of the packing in the modified atmosphere (second stage), through the analysis of ANOVA, and the Tuckey's comparing proofs in a significant level of $5 \%$.

The treatment of mango's slices with browning inhibitor agents, allowed to increase the shelf life of the product until nine days, specially the ones treated with ascorbic acid in concentration $500 \mathrm{ppm}$, due to the constant reduction of the lost of weight and excellent approval related to 
the sensorial characteristics like colour, flavor, texture and general appearance. The mango slices treated with ascorbic acid in 500 ppm and storage in modified atmosphere diminishes notoriously its lost of weight, allowing the evolution of its sugars, specially the ones in conditioned polystyrene trays and packed in bags of polyethylene-low density calibre two, it also presented the best sensorial characteristics.

Keys words: browning, mango, modified atmosphere, ascorbic acid.

Recibido: septiembre de 2007

Aprobado: diciembre de 2007

\section{Introducción}

La creciente demanda por frutas y vegetales frescos ha hecho necesario que los centros de investigación en tecnología de alimentos apunten a desarrollar técnicas de conservación y de transformación de productos agrícolas de modo que estos tengan características muy similares a las de los frescos, además de conservar sus atributos de calidad e inocuidad. Los productos minimamente procesados o de IV gama, pueden ser una solución viable.

Cuando el mango se pela y se corta para obtener un producto mínimamente procesado, presenta pardeamiento superficial en pocas horas, afectando su apariencia, lo cual influye en la calidad. Este hecho y la demanda por esta variedad en el mercado internacional, motivó el desarrollo de la presente investigación.

Los productos minimamente procesados, son aquellos a los cuales se les ha realizado una o varias operaciones unitarias tales como pelar, cortar, en combinación con técnicas de conservación como tratamiento térmico mínimo, el empleo de preservantes, aplicación de agentes antioxidantes, inmersión en agua clorada, aplicación de la técnica de atmósferas modificadas o una combinación de varias de éstas, con el fin de controlar las características físicas, químicas y biológicas de modo que su aspecto sea muy similar al de los productos frescos (Wiley, 1995; Cantwell, 1995).

Los productos minimamente procesados, permitirán a cadenas de mercados y restaurantes, obtener y conservar materia prima lista para la elaboración de productos de pastelería, pizzería, ensaladas, entre otros (Segall, et al, 1996; Catalá, et al, 2001).

Los productos mínimamente procesados presentan mayor grado de perecibilidad que los productos frescos de los que provienen (Rolle y Chism, 1987). La rotura del tejido vegetal por el corte incrementa la respiración y transpiración que conduce a un rápido deterioro del producto (Masih et al, 2002).

La presentación en rodajas, trozos, etc., es amplia, dependiendo de la naturaleza del producto no procesado y de su forma general de consumo (Huxsol y Bolin, 1989). 
El empaque en atmósfera modificada es un proceso dinámico en el que el recipiente y el producto interactúan entre sí haciendo que el ambiente gaseoso interno alcance un equilibrio adecuado que reduce la velocidad de respiración, la sensibilidad al etileno y la pérdida de humedad (Gunes, et al, 1997).

El objetivo del presente trabajo fue estudiar el efecto de la aplicación de inhibidores de pardeamiento en combinación con la técnica de atmósfera modificada sobre la calidad y tiempo de vida útil del mango variedad Van Dyke mínimamente procesado.

\section{Metodología}

Se emplearon mangos de la variedad Van Dyke provenientes de la finca Frutol ubicada en el municipio de El Espinal (Tolima). Los frutos fueron cosechados en grado de madurez 4, (más del $60 \%$ de la pulpa presentaba color amarillo).

Frutos de excelente calidad fueron transportados el mismo día de la cosecha, hasta las instalaciones de la Planta Piloto de Vegetales del Instituto de Ciencia y Tecnología de Alimentos, ICTA, de la Universidad Nacional de Colombia, sede Bogotá.

Los frutos seleccionados se lavaron y desinfectaron en solución de Mertec (200 ppm). Posteriormente se les retiró la corteza, la pulpa se troceó manualmente en cubos de $2 \mathrm{~cm}$ de arista. Los trozos se sumergieron en las soluciones de inhibidores de pardeamiento durante 10 minutos. Posteriormente, se secaron con papel absorbente y se colocaron en recipientes plásticos, los cuales fueron recubiertos con películas de polietileno de baja densidad, calibre 2 (0.035 $\mathrm{mm}$ de espesor), y se almacenaron en cuarto de refrigeración (temperatura $3 \pm 1{ }^{\circ} \mathrm{C}$, humedad relativa $85-90 \%$ ) por un tiempo de 9 días.

Una vez determinado el mejor tratamiento con inhibidores de pardeamiento, se realizó una segunda etapa aplicando el mejor inhibidor obtenido en la primera etapa con su respectiva concentración almacenando trozos de mango en condiciones de atmósfera modificada $\left(5 \% \mathrm{O}_{2}\right.$, $5 \% \mathrm{CO}_{2}, 90 \% \mathrm{~N}_{2}$ ) en dos tipos de empaque: canastilla plástica sin tapa y bandeja de icopor, los cuales fueron posteriormente introducidos, por separado, dentro de bolsas de polietileno calibre 2, durante 12 días.

Frutos procesados pero no tratados con inhibidor de pardeamiento y almacenados en atmósfera modificada, fueron tomados como testigo en las mismas condiciones de empaque y condiciones de almacenamiento.

La Tabla 1, presenta los tratamientos aplicados a los trozos de mango en las dos etapas. Las variables medidas fueron: 1) Perdida de peso, se utilizó una balanza Scout - Pro de lectura digital. 2) Sólidos solubles, fueron medidos con un refractómetro marca Brixco, 3) pH, se utilizó un pH-metro marca Metrohm 4) Contenido de azúcares (sacarosa, glucosa y fructosa) se utilizó una columna marca Alltech de intercambio catiónico, la detección de los azúcares fue hecha a través de un detector refractométrico diferencial marca Waters ${ }^{\circledR}$ Las anteriores determinaciones 
fueron realizadas cada 3 días. 5) Al final de los respectivos períodos de almacenamiento (9 días en la primera etapa y 12 días en la segunda), se realizó la evaluación sensorial sobre frutos de cada uno de los tratamientos. Fueron empleados 6 panelistas entrenados y se evaluó el aroma, el sabor, la textura, la apariencia y el color. Para el análisis estadístico de la evaluación sensorial se utilizó el método estadístico Kruscal-Wallis.

Tabla 1. Tratamientos aplicados a trozos de mango variedad Van Dyke minimamente procesados en las dos etapas

\begin{tabular}{|c|l|}
\hline TRATAMIENTO & \multicolumn{1}{c|}{ INHIBIDORES } \\
\hline Etapa 1 & \\
\hline T1 & Acido Cítrico $150 \mathrm{ppm}$ \\
\hline T2 & Acido Cítrico $300 \mathrm{ppm}$ \\
\hline T3 & Acido Ascórbico $250 \mathrm{ppm}$ \\
\hline T4 & Acido Ascórbico $500 \mathrm{ppm}$ \\
\hline T5 & L-Cysteína $100 \mathrm{ppm}$ \\
\hline T6 & L-Cysteína $200 \mathrm{ppm}$ \\
\hline Testigo & Sin inhibidor \\
\hline TRATAMIENTO & EMPAQUE E INHIBIDOR \\
\hline Etapa 2 & \\
\hline T1 & Bandeja de icopor con inhibidor \\
\hline T2 & Bandeja de icopor sin inhibidor \\
\hline T3 & Canastilla plástica con inhibidor \\
\hline T4 & Canastilla plástica sin inhibidor \\
\hline
\end{tabular}

El equipo empleado para empacar e inyectar la mezcla de gases al producto ya procesado y dispuesto en sus respectivos recipientes, fue una empacadora marca Minipack Torre SpA. Food division, serie 24044. Dalmine (BG).

A todas las variables medidas se les realizó análisis de varianza y las medias de las mediciones en cada tratamiento fueron comparadas a través de la prueba de Tuckey con una probabilidad del $95 \%$. Fueron utilizados 250 gramos por empaque y la media de 3 repeticiones fue utilizada como unidad.

Se utilizó un diseño experimental completamente al azar con arreglo factorial 3 x 2 (3 inhibidores, 2 concentraciones) para la primera etapa y 2 × 2 (2 tipos de empaque, 2 niveles de inhibidor) para la segunda etapa.

\section{Resultados y Discusión}

\section{Primera etapa}

Pérdida de peso:

Las pérdidas de peso se presentaron a partir de las 24 horas de almacenamiento en los trozos de mango de todos los tratamientos y ella fue en aumento hasta el final del almacenamiento (Figura 1).

Las menores pérdidas las registraron los frutos tratados con ácido cítrico en concentración de $300 \mathrm{ppm}$, con un valor de $6.9 \%$, seguidos por los frutos tratados con L-Cysteina a $100 \mathrm{ppm}$ 
(7.8\%), ácido ascórbico a 250 ppm (8.4\%), L-Cysteína a 200 ppm (8.7\%), ácido cítrico a 150 ppm $(9.1 \%)$, ácido ascórbico a 500 ppm $(9.8 \%)$ y finalmente los frutos testigos con un valor de $11.8 \%$.

El análisis de varianza mostró que se presentaron diferencias altamente significativas entre tratamientos al final del almacenamiento. La Prueba de comparación de Tuckey indica que no hubo diferencias entre los tratamientos con ácido cítrico (300 ppm), L-Cysteína (100 ppm) y ácido cítrico (150 ppm), ni entre los tratamientos con ácido ascórbico (250ppm) y L-Cysteína (200 ppm). Por el contrario el tratamiento con ácido ascórbico (500 ppm) y el testigo presentaron diferencias significativas entre si y con el resto de tratamientos.

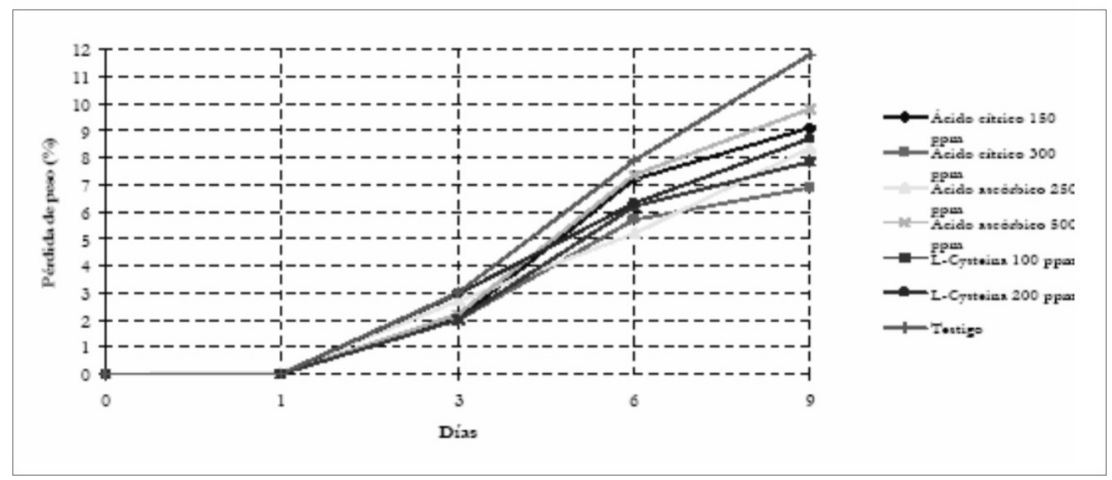

Figura 1. Porcentaje de pérdidas de peso en trozos de mango de la variedad Van Dyke tratados con diferentes concentraciones de antioxidantes

Sólidos solubles (SS)

Los sólidos solubles aumentaron en los trozos de mango de todos los tratamientos durante el periodo de almacenamiento.

La figura 2 presenta la variación de los ${ }^{\circ}$ Brix (SS), en función del tiempo de almacenamiento del producto; se observa que los frutos del tratamiento testigo presentaron a partir del tercer día, valores de ${ }^{\circ}$ Brix superiores a los trozos de fruto de los demás tratamientos. Este comportamiento se mantuvo hasta el final del almacenamiento y fue similar al comportamiento presentado por mangos de la misma variedad almacenados en atmósfera modificada (Galvis, 2005). Este comportamiento puede ser debido al efecto retardante de los inhibidores de pardeamiento en la maduración.

El Anova mostró que se presentaron diferencias altamente significativas entre los frutos de los diferentes tratamientos a lo largo del almacenamiento; la prueba de comparación de Tuckey mostró diferencias significativas al final del almacenamiento entre los frutos testigo y los frutos de los demás tratamientos. 
$\mathrm{pH}$

El pH de los frutos de todos los tratamientos presentó tendencia a aumentar hasta el día 6 de almacenamiento; sin embargo los frutos tratados con L-Cysteína (100 y 200 ppm) y los tratados tratamientos el pH de los trozos presentó aumento hasta el final del almacenamiento, indicando que en ellos el proceso de maduración continuó (Tovar et al, 2001).

La Figura 3 presenta el comportamiento de los trozos de mango respecto al $\mathrm{pH}$ en los diferentes tratamientos con inhibidores.

El análisis de varianza muestra que el $\mathrm{pH}$ presentó diferencias altamente significativas entre los trozos de los diferentes tratamientos a lo largo del almacenamiento. La prueba de comparación de Tuckey mostró para el día 9 que no hubo diferencias significativas entre los trozos tratados con ácido cítrico (150 ppm) y ácido ascórbico (500 ppm); sin embargo estos presentaron diferencias significativas con todos los frutos de los demás tratamientos.

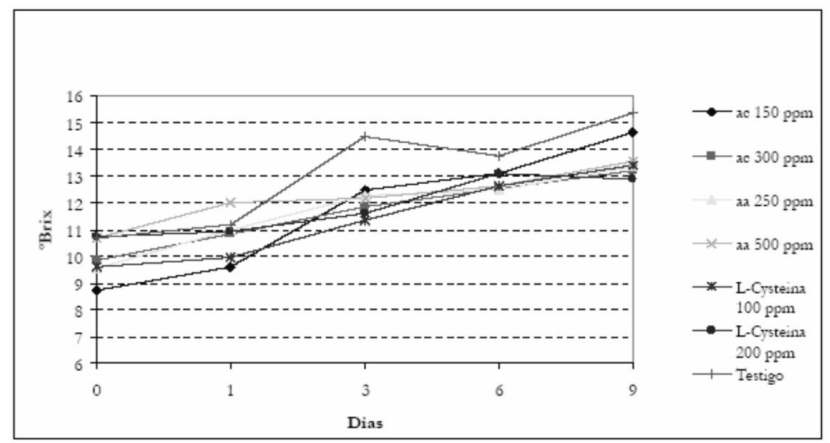

Figura 2. Variación de los SS ( ${ }^{\circ}$ Brix) en trozos de mango de la variedad Van Dyke tratados con diferentes concentraciones de antioxidantes

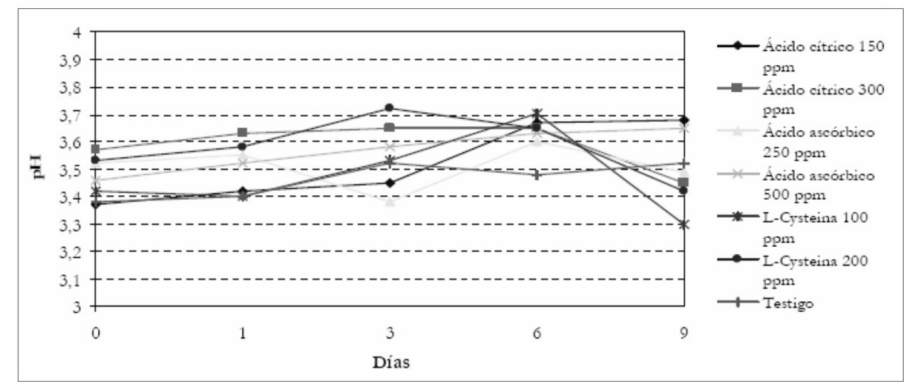

Figura 3. Variación del pH en trozos de mango de la variedad Van Dyke tratados con diferentes concentraciones de antioxidantes 


\section{Análisis sensorial}

En la figura 4, se observa que los trozos de fruta del tratamiento con ácido ascórbico en concentración de 500 ppm (aa 500) presentaron las mejores calificaciones respecto al color, sabor, aroma, textura y apariencia general, seguidos de los frutos de los tratamientos con ácido cítrico en concentración de 150 ppm (ac 150), los frutos testigo, los frutos del tratamiento con ácido ascórbico en concentración de 250 ppm (aa 250) y los trozos tratados con L-cysteina en concentración de 200 ppm (L-200). Los frutos tratados con L-Cysteína en concentración de 100 ppm obtuvieron las calificaciones más bajas.

El análisis estadístico de Kruscal - Wallis para el día 9 de almacenamiento mostró que se presentaron diferencias significativas en las características sensoriales evaluadas en los frutos de los tratamientos.

De acuerdo a los resultados obtenidos con relación a las propiedades físicas, químicas y especialmente las sensoriales de los frutos de los diferentes tratamientos, como también el análisis estadístico, se estableció que la mejor respuesta en condiciones de calidad en los 9 días de almacenamiento, la presentaron los trozos de mango tratados con ácido ascórbico en concentración de 500 ppm.

En este tratamiento los trozos de mango obtuvieron las más altas calificaciones en el panel sensorial en las cualidades de color, sabor, textura y apariencia general.

Por lo anterior este tratamiento fue escogido para ser aplicado en trozos de mango en combinación con la técnica de atmósfera modificada durante 12 días de almacenamiento empleando dos tipos de empaque como se mencionó en la metodología.

Aunque los resultados del análisis microbiológico no se presentan en este artículo, los trozos de mango tratados con esta solución, obtuvieron los más bajos recuentos de mohos, levaduras y coliformes totales por debajo de 3 y los recuentos de esporas clostridium sulfito reductoras por debajo de 10 hasta el final del periodo de almacenamiento.

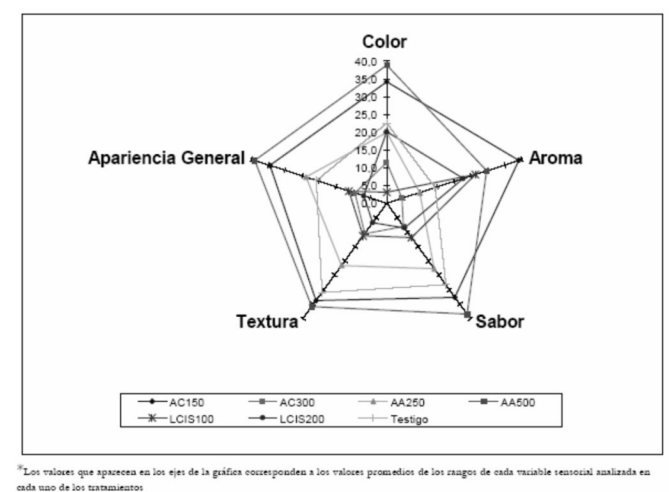

Figura 4. Evaluación sensorial de trozos de mango variedad Van Dyke tratado con antioxidantes en dos concentraciones al final del almacenamiento 


\section{Segunda etapa}

Al igual que en la primera fase del estudio, para establecer el efecto de la aplicación de ácido ascórbico en concentración de 500 ppm, como inhibidor de pardeamiento en trozos de mango en combinación con la técnica de atmósfera modificada, se observaron los cambios en el tiempo, de las propiedades físicas, químicas, y sensoriales del producto.

Galvis et al (2005) trabajando con frutos enteros de esta misma variedad acondicionados en AM dentro de bolsas de polietileno de baja densidad calibre 2 ( $0.035 \mathrm{~mm}$ de espesor), encontraron que los mejores resultados se presentaron para una composición inicial de gases de $5 \%$ de $\mathrm{O}_{2}, 5 \%$ de $\mathrm{CO}_{2}$ y $90 \%$ de $\mathrm{N}_{2}$, debido a que permitió la maduración de las frutas sin que se presentaran alteraciones en sus características organolépticas en un tiempo mayor respecto a los frutos testigo, colocados a una temperatura de $12^{\circ} \mathrm{C}$.

Pérdida de peso

Como se observa en la figura 5, los trozos de mango del tratamiento testigo, empacados en bandeja de icopor, presentaron la mayor pérdida de peso durante el almacenamiento, con un valor de $4.0 \%$, seguido del producto tratado con ácido ascórbico en 500 ppm empacado en bandejas de icopor con $3.4 \%$. La menor pérdida de peso la registraron los trozos de mango del tratamiento con ácido ascórbico en 500 ppm y los del tratamiento testigo empacado en canastilla plástica, con $1.8 \%$ y $2.6 \% \%$ respectivamente.

Comparando la variación de peso de los trozos de mango en estos tratamientos se observa que los acondicionados en canastilla plástica, registraron menores variaciones de peso, lo cual permite concluir que el tipo de empaque influye altamente en la variación de peso del producto.

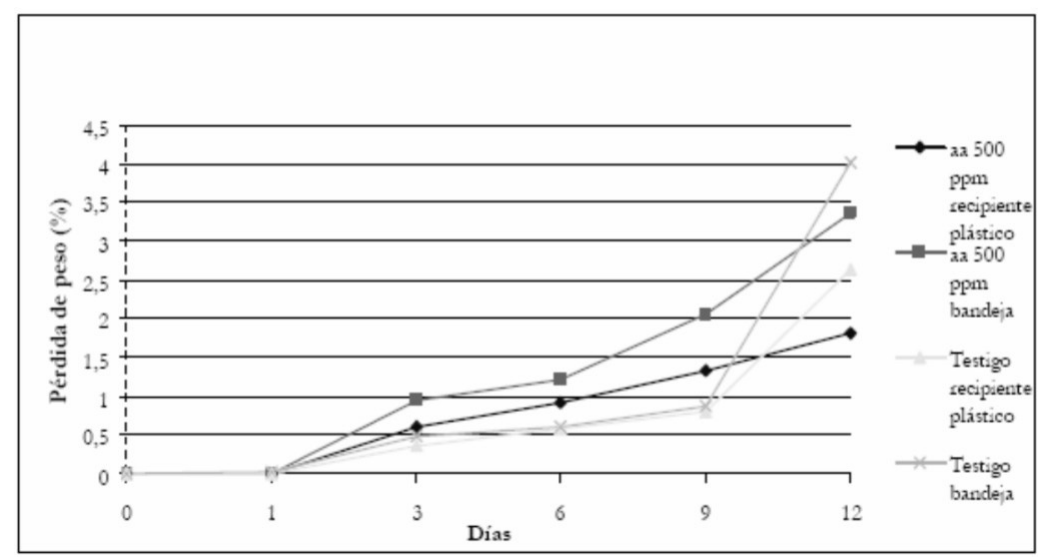

Figura 5. Porcentaje de pérdidas de peso en trozos de mango de la variedad Van Dyke en atmósfera modificada tratados con ácido ascórbico 500 ppm, en dos condiciones de empaque 
El Anova mostró que existieron diferencias altamente significativas entre los frutos de los tratamientos a partir del día 6 hasta el final del almacenamiento, pero no se encontraron diferencias en las condiciones de empaque empleadas en el mismo periodo de tiempo. Sin embargo, es importante anotar que las pérdidas de peso observadas en trozos de mango variedad Van Dyke almacenados en AM fueron menores que las que se registraron en la misma variedad empacados en atmósfera normal (primera etapa) y en las mismas condiciones hasta el noveno día de almacenamiento.

Fonseca (2005), observó que en mango minimamente procesado de la variedad keit y empacado en atmósfera modificada, la pérdida de peso fue de $26 \%$ en frutos testigo, del $18 \%$ para mangos troceados empacados en películas plásticas de baja densidad calibres 2 y 3 y del $15.5 \%$ en trozos empacados en canastillas plásticas, almacenados a $12^{\circ} \mathrm{C}$. Estas diferencias muestran la influencia de la temperatura en la conservación del producto.

$\mathrm{pH}$

El pH de los trozos de mango de todos los tratamientos mostró un aumento hasta el noveno día y luego una disminución hasta finalizar el periodo de almacenamiento (Figura 6). Senesi et al, (1999), encontraron un comportamiento diferente en peras mínimamente procesadas, las cuales presentaron un leve aumento en los últimos tres días de almacenamiento tanto en las tratadas con antioxidantes como en aquellas tomadas como testigo.

El análisis de varianza muestra que se presentaron diferencias altamente significativas en el valor de $\mathrm{pH}$ de los trozos de mango de todos los tratamientos durante el periodo de almacenamiento y la prueba de comparación de Tuckey indica que hay diferencias significativas entre los trozos de fruta tratados con inhibidor y los no tratados tomados como testigo a partir del tercer día de almacenamiento, así como entre las condiciones de empaque empleadas, lo cual significa que las condiciones de empaque influyen sensiblemente en el valor de $\mathrm{pH}$.

\section{Azúcares}

\section{Sacarosa}

La sacarosa fue el azúcar que presentó los niveles más altos en todos los tratamientos en atmósfera modificada, comparado con fructosa y glucosa como se observa en la tabla 2. Promedios con la misma letra leídos verticalmente no presentan diferencia significativa, según la prueba de Tuckey al 5\% de significancia. 


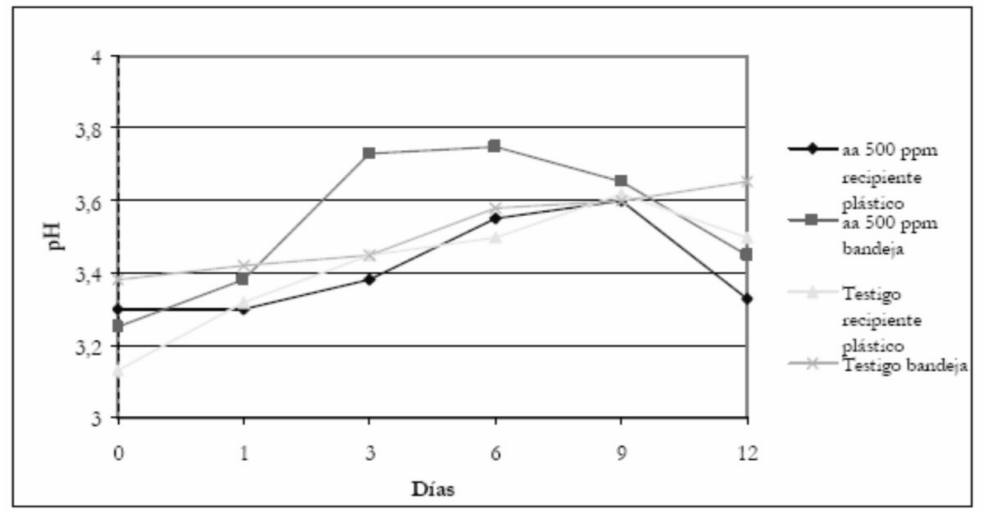

Figura 6. Variación del pH en trozos de mango de la variedad Van Dyke en atmósfera modificada tratadas con ácido ascórbico 500 ppm, en dos condiciones de empaque

Tabla 2. Valores promedio de azúcares de trozos de mango variedad Van Dyke enpacados en atmósferas modificada $(\mathrm{mg} / \mathrm{g})$

\begin{tabular}{|l|c|c|c|}
\hline \multirow{2}{*}{ TRATAMIENTOS } & \multicolumn{3}{|c|}{ TIEMPO (DÍAS) } \\
\cline { 2 - 4 } & $\mathbf{0}$ & $\mathbf{6}$ & $\mathbf{1 2}$ \\
\hline AA Canastilla & & & \\
\hline Sacarosa & $5.36 \mathrm{~b}$ & $5.29 \mathrm{~b}$ & $6.25 \mathrm{a}$ \\
\hline Glucosa & $0.41 \mathrm{c}$ & $0.53 \mathrm{~b}$ & $0.77 \mathrm{~b}$ \\
\hline Fructosa & $1.87 \mathrm{c}$ & $2.05 \mathrm{~b}$ & $2.71 \mathrm{a}$ \\
\hline AA Bandeja & & & \\
\hline Sacarosa & $5.97 \mathrm{~b}$ & $5.40 \mathrm{~b}$ & $6.02 \mathrm{ab}$ \\
\hline Glucosa & $0.50 \mathrm{~b}$ & $0.68 \mathrm{a}$ & $0.75 \mathrm{~b}$ \\
\hline Fructosa & $2.12 \mathrm{~b}$ & $2.21 \mathrm{a}$ & $268^{\mathrm{a}}$ \\
\hline Testigo Canastilla & & & \\
\hline Sacarosa & $7.73 \mathrm{a}$ & $6.28 \mathrm{a}$ & $5.52 \mathrm{~b}$ \\
\hline Glucosa & $0.58 \mathrm{a}$ & $0.68 \mathrm{a}$ & $0.80^{\mathrm{a}}$ \\
\hline Fructosa & $2.74 \mathrm{a}$ & $2.16 \mathrm{a}$ & $2.46 \mathrm{~b}$ \\
\hline Testigo Bandeja & & & \\
\hline Sacarosa & $6.11 \mathrm{a}$ & $6.89 \mathrm{a}$ & $6.67 \mathrm{a}$ \\
\hline Glucosa & $0.51 \mathrm{~b}$ & $0.58 \mathrm{~b}$ & $0.78 \mathrm{~b}$ \\
\hline Fructosa & $1.56 \mathrm{c}$ & $2.09 \mathrm{~b}$ & $2.03 \mathrm{c}$ \\
\hline
\end{tabular}

El análisis de varianza mostró que hubo diferencias altamente significativas en el contenido de sacarosa de los frutos entre tratamientos, la prueba de Tuckey mostró que los trozos de mango provenientes del tratamiento en bandeja de icopor presentaron valores similares en el contenido de sacarosa a lo largo del almacenamiento, igual comportamiento presentaron los frutos provenientes del tratamiento con ácido ascórbico en bandeja de icopor.

\section{Glucosa}

La Tabla 2 muestra que este azúcar presentó el menor contenido en todos los tratamientos, durante el periodo de almacenamiento. Este hecho es importante debido a que en la glucosa actúan proteínas enzimáticas que participan en reacciones redox (oxidación), lo que puede dar 
lugar a pardeamiento (Salisbury, 1994). Además la glucosa es un azúcar que participa en el proceso de respiración reduciendo su contenido (Arjona, et al, 2000)

El Anova mostró que hubo diferencias altamente significativas entre tratamientos. La prueba de Tuckey mostró diferencias en el contenido de glucosa entre los trozos de mango tratados con ácido ascórbico y aquellos que fueron tomados como testigo, los cuales presentaron los mayores contenidos durante el almacenamiento tanto en canastilla plástica como en bandeja de icopor, (tabla 2). Un comportamiento similar fue observado por Gálvis et al (2005), en mangos enteros de la misma variedad almacenados en diferentes condiciones de $\mathrm{AM} \mathrm{a} 12^{\circ} \mathrm{C}$, durante 25 días de almacenamiento, siendo superior en los frutos del tratamiento testigo a aquellos acondicionados en bolsas calibre 2 .

\section{Fructosa}

Este azúcar se encontró en niveles más bajos que los de sacarosa, pero más altos que los de glucosa. Este comportamiento se presentó en todos los tratamientos a lo largo del tiempo de almacenamiento. En la tabla 2, se observa que la fructosa aumentó hasta el final del ensayo en los trozos de mango de los tratamientos con ácido ascórbico, además de presentar los mayores valores, siendo más elevado en los trozos empacados en canastilla plástica que en los colocados en bandeja de icopor. Por el contrario, los trozos de los tratamientos testigo colocados en bandejas de icopor, registraron los menores contenidos de fructosa. Senesi, et al (1999), observaron que en peras troceadas de las variedades Abate Fetel y Kaiser la fructosa disminuye durante 12 días y aumenta en los últimos 3 días de almacenamiento. Al igual que para la sacarosa la prueba de comparación de Tuckey mostró que no hay diferencias significativas entre los trozos de mango tratados con ácido ascórbico y los tomados como testigo para este azúcar.

\section{Análisis sensorial}

En el análisis de las características sensoriales se encontraron diferencias significativas de las variables evaluadas en los trozos de mango de los diferentes tratamientos al final del almacenamiento, como se muestra en la figura 7.

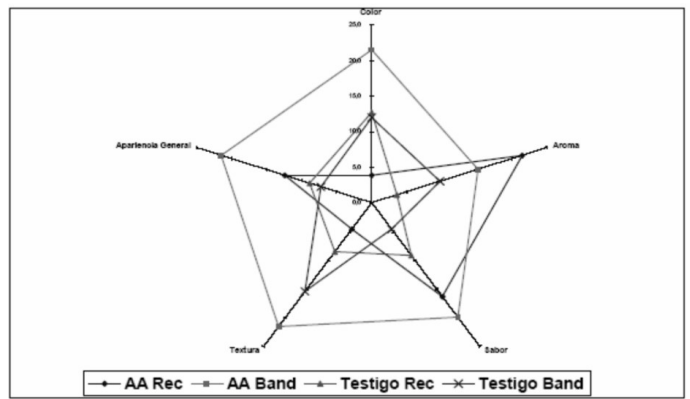

Figura 7. Evaluación sensorial de mango variedad Van Dyke mínimamente procesado, al final del almacenamiento, tratado con ácido ascórbico (500ppm) en atmósfera modificada 
La mejor respuesta respecto al color, la apariencia general, la textura y el sabor lo presentaron los trozos del tratamiento con ácido ascórbico empacado en bandeja de icopor (AA Band) y los que recibieron la más baja calificación fueron los trozos del tratamiento con ácido ascórbico empacado en canastilla plástica (AA Rec) y los frutos testigo empacados en bandeja de icopor, lo cual resalta el efecto benéfico del ácido ascórbico como inhibidor de pardeamiento y la canastilla plástica como empaque.

Brecht (1995), afirma que la tolerancia de frutas y vegetales a niveles bajos de $\mathrm{O}_{2}$ y elevadas concentraciones de $\mathrm{CO}_{2}$, la mayoría de las veces, es determinante en la prolongación de la vida útil de frutas y vegetales mínimamente procesados, dado que reduce e inhibe la actividad metabólica, especialmente la acción y biosíntesis del etileno. Este mismo autor sostiene que el monóxido de carbono es algunas veces, incluido para inhibir el pardeamiento y el crecimiento de microorganismos y este efecto pudo ser la razón por la que en los trozos de mango tratados con ácido ascórbico en concentración 500 ppm y acondicionados en bandeja de icopor hayan tenido menores recuentos de mohos y levaduras, así como de mesófilos aerobios.

\section{Conclusiones}

- Los resultados de la primera etapa de la investigación muestran que mediante la aplicación de la tecnología de alimentos minimamente procesados, es posible prolongar la vida útil de mango troceado de la variedad Van Dyke hasta por 9 días de almacenamiento a $3 \pm 1^{\circ} \mathrm{C}$ colocados en atmósfera normal.

- El antioxidante aplicado al mango de la variedad Van Dyke minimamente procesado acondicionado en atmósfera normal, que mejor efecto causó fue el ácido ascórbico en concentración de 500 ppm.

- El análisis sensorial realizado en los trozos de mango tratados con ácido ascórbico (500 ppm), empacados en canastilla plástica en atmósfera normal y los tratados con el mismo inhibidor en atmósfera modificada (AM) en las mismas condiciones de almacenamiento, mostró que este último tratamiento es más efectivo en la conservación del mango.

- Los trozos de mango en AM que obtuvieron las calificaciones más altas en la evaluación sensorial respecto al color, sabor, textura y apariencia general, fueron los tratados con ácido ascórbico en 500 ppm, colocados en bandejas de icopor e introducidos en bolsas de polietileno de baja densidad calibre 2 .

- El ácido ascórbico en 500 ppm demostró ser un inhibidor de pardeamiento efectivo en las dos etapas del presente estudio. 


\section{Referencia Bibliográficas}

ARJONA, H., GALVIS, J. A. 2000. Influencia de la temperatura y el tiempo de almacenamiento en la conservación del mango (Manguífera índica L) variedad Van Dyke. En: 2do. Congreso Iberoamericano de Tecnología Poscosecha y Agroexportaciones. Bogotá Colombia.

BRECHT, J. 1995. Physiology of lightly processed fruit and vegetables. Horticultural Sciences Department. University of Florida. En: HortScience. Alexandria. Vol 30 No 1 (Feb); p18

FONSECA, L. 2005. Obtención y conservación de mango (Mangífera Indica L) mínimamente procesado variedad keitt. Universidad Nacional de Colombia. Facultad de Ingeniería. Departamento de Ingeniería Civil y Agrícola. Bogotá.

GALVIS, J. A, ARJONA H. FISCHER G AND MARTINEZ R. 2005. Using modified atmosphere packaging for storing 'Van Dyke' mango (Mangífera Índica, L) fruit. En: Revista Agronomía Colombiana, Volumen XXIII Nº 2, ISSN 0120-9965, Bogotá

GALVIS, J. A. 2002. Análisis del crecimiento del fruto de mango (Mangífera Índica, L) variedad Van Dyke y estudio de algunos métodos de conservación poscosecha. Universidad Nacional de Colombia. Facultad de Agronomía, Tesis Doctoral. Bogotá.

GUNES, G., Y LEE, CHANG Y. 1997. Color of minimally processed potatoes modified atmosphere packaging and anti-browning agents. Journal of Food Science and Technology International-volume 62,No 3: 572-575

HUXSOLL C. C., BOLIN H. R. 1989. Processing and distribution alternatives for minimally processed fruit and vegetables. Food Technology. 43(2), 123.

MASIH, L., ROGINSKI, H., PREMIER, R., TOMKINS, B. AND AJLOUNI, S. 2002. Soluble protein content in minimally processed vegetables during storage. Journal of Food Science and Technology International. 35 (2002) 697-702

ROLLE, R. S. Y CHISM, G. W. 1987. Physiological consequences of minimally processed fruits and vegetables. Journal Food Qual. 10: 157-177

SALISBURY, F. Y ROSS, C. 1994. Fisiología vegetal. Editorial Iberoamericana. México.

SEGALL, K. Y SCANLON, M. 1996. Design and Analysis of a Modified-atmosphere Package for Minimally Processed Romaine Lettuce. Journal American Society HortScience 121(4): 722-729 
SENESI, E., GALVIS, J., AND FUMAGALLI, G. 1999. Quality indexes and internal atmosphere of packaged fresh-cut pears Abatate Fetel and Kaiser varieties. Journal of Food Science and Technology International 2: 111-120.

TOVAR, B., GARCÍA, H. Y MATTA, M. 2001. Journal Food Research International. 34 (2001) 705-714

WILEY, R. C. 1997. Frutas y hortalizas mínimamente procesadas y refrigeradas. Madrid. España. Editorial acriba, 362p 